A. Szankowski

\title{
Three-space problems for the approximation property
}

Received February 29, 2008

Abstract. It is shown that there is a subspace $Z_{q}$ of $\ell_{q}$ for $1<q<2$ which is isomorphic to $\ell_{q}$ and such that $\ell_{q} / Z_{q}$ does not have the approximation property (AP). On the other hand, for $2<p<\infty$ there is a subspace $Y_{p}$ of $\ell_{p}$ such that $Y_{p}$ does not have AP but $\ell_{p} / Y_{p}$ is isomorphic to $\ell_{p}$. The result is obtained by defining random "Enflo-Davie spaces" $Y_{p}$ which with full probability fail to have AP for all $2<p \leq \infty$ and have AP for all $1 \leq p \leq 2$. For $1<p \leq 2, Y_{p}$ is isomorphic to $\ell_{p}$.

Keywords. Quotients of Banach spaces, approximation property

In this paper we prove the result stated in the abstract. In particular, it solves the following problem which has been around since the 1970's:

(\$) Does there exist a reflexive Banach space $X$ and a subspace $Y \subset X$ such that both $X$ and $Y$ have $A P$ but $X / Y$ does not have AP?

Let us recall that a Banach space $X$ is said to have the approximation property (AP) if for every compact set $K$ in $X$ and for every $\varepsilon>0$, there is a finite rank operator $T=T_{K}$ on $X$ such that $\|T x-x\| \leq \varepsilon$ for every $x \in K$. When all these $T_{K}$ 's are uniformly bounded, we say that $X$ has the bounded approximation property (BAP). If a Banach space has a (Schauder) basis, then it has BAP.

In 1972 P. Enflo gave the first example of a Banach space without AP.

A thorough discussion of approximation properties can be found in [4].

The problem (\$) is a sort of a "three space problem". These are problems of the following type:

Let $X, Y, Z$ be Banach spaces with $Y \subset X$ and $Z=X / Y$, or putting it in a fancier way, let $0 \rightarrow Y \rightarrow X \rightarrow Z \rightarrow 0$ be a short exact sequence. Suppose that two of the spaces $X, Y, Z$ have a certain property $\mathcal{P}$. Does this imply that the third one has $\mathcal{P}$ ?

Thus for every property $\mathcal{P}$ we have three different three-space problems; let us call them, respectively, the $X, Y, Z$-problems (e.g., if $X, Y$ have $\mathcal{P}$, we have the $Z$-problem, etc.).

A. Szankowski: Institute of Mathematics, The Hebrew University, Jerusalem, Israel; e-mail: tomek@math.huji.ac.il

Mathematics Subject Classification (2000): Primary 46B20, 46B07; Secondary 46B99 
In the context of AP these three problems are mutually inequivalent and none is trivial:

(i) By [2], every separable Banach space $X$ contains a subspace $Y$ such that both $Y$ and $X / Y$ have BAP. Taking for $X$ a separable Banach space without AP, we obtain a counterexample to the $X$-problem.

(ii) It follows from our result that the $Y$-problem has a negative solution, i.e. there exist $X$ with AP and $Y \subset X$ without AP so that $X / Y$ has AP. We do not know whether this follows from older results.

(iii) By [3], for every separable Banach space $Z$ there is a Banach space $Y$ with basis such that $Y^{* *}$ has a basis and $Y^{* *} / Y$ is isomorphic to $Z$. Taking for $Z$ a separable space without AP, we obtain a counterexample to the $Z$-problem. Let us observe that one cannot dualize this example in order to obtain a negative solution to the $Y$-problem.

Evidently, in (iii) $X=Y^{* *}$ is inherently a nonreflexive space and this approach is unlikely to provide a reflexive example. This makes the question (\$) quite natural.

To prove our main result (Theorem 2 we of course use the Enflo-Davie machinery for constructing subspaces of $\ell_{p}, p>2$, without AP. The most important new tool is Theorem 3, which is a delicate refinement of Kashin's splitting theorem and quite likely will have other applications.

\section{Preliminaries}

\section{Some notation}

For $\alpha=(\alpha(j))_{j=1}^{n} \in \mathbb{R}^{n}$ and $1 \leq s \leq \infty$ we define

$$
|\alpha|_{s}=\left(n^{-1} \sum_{j=1}^{n}|\alpha(j)|^{s}\right)^{1 / s} \quad\left(|\alpha|_{\infty}=\max |\alpha(j)|\right)
$$

and for $\alpha, \beta \in \mathbb{R}^{n}$ we define

$$
\langle\alpha, \beta\rangle=n^{-1} \sum_{j=1}^{n} \alpha(j) \beta(j) .
$$

Let $X$ denote the (real) vector space of all sequences $a=\left(a_{k}\right)_{k=0}^{\infty}$ with $a_{k} \in \mathbb{R}^{3 \cdot 2^{k}}$ and $a_{k}=0$ for almost all $k$. For $a, b \in X$ we define

$$
\langle a, b\rangle=\sum_{k=0}^{\infty}\left\langle a_{k}, b_{k}\right\rangle .
$$

The norms $\|\cdot\|_{s, p}$ on $X$ are defined by

$$
\left\|\left(a_{k}\right)_{k=0}^{\infty}\right\|_{s, p}=\left(\sum_{k=0}^{\infty}\left|a_{k}\right|_{s}^{p}\right)^{1 / p} .
$$


We shall identify $\mathbb{R}^{3 \cdot 2^{k}}$ with the subspace $\left\{(0, \ldots, u, 0, \ldots): u \in \mathbb{R}^{3 \cdot 2^{k}}\right\}$ of $X$. Notice that if $u \in \mathbb{R}^{3 \cdot 2^{k}}$, then $\|u\|_{s, p}=|u|_{s}$ for every $s, p$.

Let $X_{s, p}$ be the completion of $X$ in the norm $\|\cdot\|_{s, p}$. It is obvious that $X_{p, p}$ is isometric to $\ell_{p}$. By Pełczyński's decomposition method (cf. [4, p. 54]), we know that $X_{2, p}$ is isomorphic to $\ell_{p}$ for $1<p<\infty$.

Let $E \subset \mathbb{R}^{m}$ and $F \subset \mathbb{R}^{p}$ with $\operatorname{dim} F \leq \operatorname{dim} E$. Denote by $O(F, E)$ the set of all orthogonal (with respect to the above inner products in $\mathbb{R}^{m}, \mathbb{R}^{p}$, respectively) transformations from $F$ to $E$ and let $v_{F, E}$ denote the normalized invariant measure on $O(F, E)$. We

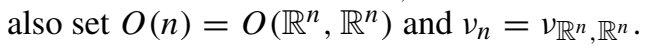

Let $E \subset \mathbb{R}^{m}$. Let $S_{E}=\left\{x \in E:|x|_{2}=1\right\}$, let $\sigma_{E}$ denote the normalized surface measure on $S_{E}$, and set $S_{m}=S_{\mathbb{R}^{m}}$ and $\sigma_{m}=\sigma_{\mathbb{R}^{m}}$.

The normalized unit vectors $e_{i}^{m} \in \mathbb{R}^{m}$ are defined by $e_{i}^{m}=m^{1 / 2}\left(\delta_{i j}\right)_{j=1}^{m}$.

Throughout this paper $C$ and $c$ are fixed positive numbers.

\section{Enflo's construction (cf. [4, Ch. 2.d])}

Let us briefly recall this classical construction, in the form due to Davie. The following Theorem 1 is a "genericized" formulation.

Given a sequence $\left\{T_{k}\right\}_{k=0}^{\infty}$ with $T_{k} \in O\left(3 \cdot 2^{k}\right)$ for $k=0,1, \ldots$, define

$$
\begin{array}{ll}
u_{i}^{k}=T_{k}^{*}\left(e_{i}^{3 \cdot 2^{k}}\right) & \text { for } i=1, \ldots, 2^{k}, \\
v_{i}^{k}=T_{k}^{*}\left(e_{2^{k}+i}^{3 \cdot 2^{k}}\right) & \text { for } i=1, \ldots, 2^{k+1}, \\
y_{i}^{k}=v_{i}^{k-1}+u_{i}^{k} & \text { and } \quad z_{i}^{k}=v_{i}^{k-1}-u_{i}^{k} \quad \text { for } i=1, \ldots, 2^{k}, k=1,2, \ldots
\end{array}
$$

Let $Y=\operatorname{span}\left\{y_{i}^{k} \in X: k=1,2, \ldots, i=1, \ldots, 2^{k}\right\}$ and let $Y_{p}=Y_{p}\left(\left\{T_{k}\right\}\right)$ be the completion of $Y$ in the norm $\|\cdot\|_{p, p}$.

Theorem 1. There is a sequence $\left\{T_{k}\right\}_{k=0}^{\infty}$ such that $Y_{p}=Y_{p}\left(\left\{T_{k}\right\}\right)$ does not have AP for all $2<p \leq \infty$. Moreover, this is a generic fact, i.e. denoting by $v$ the product measure $v=\otimes v_{3 \cdot 2^{k}}$ we have

$$
v\left(\left\{\left\{T_{k}\right\}: Y_{p}\left(\left\{T_{k}\right\}\right) \text { fails to have AP for all } 2<p \leq \infty\right\}\right)=1 .
$$

In this and in the next theorem we ignore the issues of measurability. What we really mean is that the above set contains a set of full measure.

We shall not prove this theorem; we think it belongs to the folklore of Banach space theory, although probably it has never been formulated this way. On demand, we are ready to supply the details of its proof. 


\section{Some estimates}

Let us first recall two "volumetric" estimates (cf. [6, Ch. 6, (6.3)]). For $0<r \leq 1$ define $A_{m}(r)=\left\{x \in S_{m}:|x|_{1} \leq r\right\}$. We have

$$
\sigma_{m}\left(A_{m}(r)\right) \leq(C r)^{m}
$$

and

$$
N\left(A_{m}(r), \delta\right) \leq(3 e / r)^{3 r m / \delta} .
$$

Here for $A \subset \mathbb{R}^{m}$, by $N(A, \delta)$ we denote the covering number by euclidean balls of radius $\delta$ :

$$
N(A, \delta)=\min \left\{N: \exists x_{1}, \ldots, x_{N} \in A \text { such that } A \subset \bigcup_{i=1}^{N} B\left(x_{i}, \delta\right)\right\} .
$$

Notice also that if $B$ is an $l$-dimensional euclidean unit ball, then

$$
N(B, \delta) \leq(3 / \delta)^{l}
$$

(cf. [6, Lemma 4.16]).

We shall frequently use the rotational invariance of the measures $\sigma_{E}$ and $\nu_{F, E}$. Let us here observe that for every measurable set $A \subset S_{E}$ and every $x \in S_{F}$ we have

$$
\sigma_{E}(A)=v_{F, E}(\{T \in O(F, E): T x \in A\}) .
$$

\section{The construction}

We shall prove the following theorem.

\section{Theorem 2.}

$$
v\left(\left\{\left\{T_{k}\right\}: Y_{q}\left(\left\{T_{k}\right\}\right) \text { is isomorphic to } \ell_{q} \text { for all } 1<q \leq 2\right\}\right)=1 .
$$

This, together with Theorem 1, yields all the results stated in the abstract:

With the notation of (1), let $Z=\operatorname{span}\left\{z_{i}^{k} \in X: k=1,2, \ldots, i=1, \ldots, 2^{k}\right\}$ and let $Z_{p}$ be the completion of $Z$ in the norm $\|\cdot\|_{p, p}$. Observe that the linear map $R: Y \rightarrow Z$ defined by $R\left(y_{i}^{k}\right)=(-1)^{k}\left(z_{i}^{k}\right)$ is a $\|\cdot\|_{p, p}$ isometry for all $p$, thus $Y_{p}$ and $Z_{p}$ are isometric. In particular, $Z_{p}$ fails to have AP for $2<p \leq \infty$ and is isomorphic to $\ell_{p}$ for $1<p \leq 2$ (generically).

We have the natural identification $\left(X_{p, p} / Y_{p}\right)^{*}=Y_{p}^{\perp} \subset X_{q, q}(1 / p+1 / q=1)$. We see that $X=\mathbb{R} \cdot u_{1}^{0} \oplus Y \oplus Z$ (orthogonal sum in the sense of $\langle$,$\rangle ). Therefore \left(X_{p, p} / Y_{p}\right)^{*}$ is isometric to $\mathbb{R} \cdot u_{1}^{0} \oplus Z_{q}$, hence isomorphic to $Z_{q}$.

Therefore $X_{p, p} / Y_{p}$ is isomorphic to $\ell_{p}$ for $2 \leq p<\infty$ (generically). Since AP is a self-dual property for reflexive spaces, we deduce that (generically) $X_{p, p} / Y_{p}$ fails to have AP for $1<p<2$ (and for $p=1$ as well, but this requires a slightly more delicate argument). 
Let $E$ be a subspace of $\mathbb{R}^{m}$. We define the norm $|\cdot|_{E}^{\perp}$ on $E$ by

$$
|x|_{E}^{\perp}=\min \left\{|x+y|_{1}: y \in E^{\perp}\right\} .
$$

Set $A_{E}^{\perp}(r)=\left\{x \in S_{E}:|x|_{E}^{\perp} \leq r\right\}$ (notice that $A_{\mathbb{R}^{m}}^{\perp}=A_{m}(r)$ ). It is important to realize that the estimates (2) and (3) hold for $A_{E}^{\perp}(r)$ as well:

Lemma 1. Let $E \subset \mathbb{R}^{m}$ with $n=\operatorname{dim} E \geq m / 3$, and let $0 \leq r<\delta / 2 \leq 1$ with $\delta \leq 3 r m$. Then

$$
\sigma_{E}\left(A_{E}^{\perp}(r)\right) \leq(C r)^{n}
$$

and

$$
N\left(A_{E}^{\perp}(r), \delta\right) \leq(3 e / r)^{3 r m / \delta}
$$

Proof. To prove (6), let $D$ denote the unit ball of $|\cdot|_{E}^{\perp}$, thus $D=\operatorname{absconv}\left\{m^{1 / 2} P e_{i}^{m}\right.$ : $1 \leq i \leq m\}$ where $P: \mathbb{R}^{m} \rightarrow E$ denotes the orthogonal projection. Therefore

$$
D=\bigcup_{A \subset\{1, \ldots, m\}, \# A=n} \operatorname{absconv}\left\{m^{1 / 2} P e_{i}^{m}: i \in A\right\} .
$$

By Hadamard's inequality, if \# $A=n$, then

$$
\operatorname{vol}\left(\operatorname{absconv}\left\{m^{1 / 2} P e_{i}^{m}: i \in A\right\}\right) \leq \operatorname{vol} B_{1}^{n} \leq C^{n} \operatorname{vol} B_{2}^{n}
$$

$\left(B_{p}^{n}\right.$ denotes the unit ball of $|\cdot|_{p}$ in $\mathbb{R}^{n}$ ). Therefore

$$
\operatorname{vol} D \leq C^{n}\left(\begin{array}{c}
m \\
n
\end{array}\right) \text { vol } B_{2}^{n} \leq C^{n} 2^{m} \operatorname{vol} B_{2}^{n} \leq(8 C)^{n} \operatorname{vol} B_{2}^{n},
$$

i.e. the volume ratio of $D$ is bounded by $8 C$. By (6.3) in [6], (6) holds.

The proof of (7) is a modification of a classical proof of (3) (cf. [7]): $|\cdot|_{E}^{\perp}$ is the Minkowski functional of the set $D=\operatorname{absconv}\left\{m^{1 / 2} P e_{i}^{m}: 1 \leq i \leq m\right\}$. Thus if $x \in$ $A_{E}^{\perp}(r)$, then $x=m^{1 / 2} \sum_{i=1}^{m} t_{i} P e_{i}^{m}$ with $\sum_{i=1}^{m}\left|t_{i}\right| \leq r$.

Let $\pi$ be a permutation of $\{1, \ldots, m\}$ such that $\left|t_{\pi(1)}\right| \geq\left|t_{\pi(2)}\right| \geq \cdots$. For every $1 \leq l \leq m$, we have

$$
r \geq \sum_{i=1}^{m}\left|t_{i}\right| \geq l \cdot\left|t_{\pi(l)}\right| \geq \frac{l}{\sqrt{m-l}}\left(\sum_{i \geq l} t_{\pi(i)}^{2}\right)^{1 / 2} .
$$

Let $b=m^{1 / 2} \sum_{i \geq l} t_{\pi(i)} P e_{i}^{m}=P y$ where $y=m^{1 / 2} \sum_{i \geq l} t_{\pi(i)} e_{i}^{m}$. We have, by $(8)$,

$$
|b|_{2} \leq|y|_{2}=\sqrt{m}\left(\sum_{i \geq l} t_{\pi(i)}^{2}\right)^{1 / 2} \leq \frac{r}{l} \cdot \sqrt{m(m-l)} \leq \frac{r m}{l} .
$$

For $c \in E$ we shall write $l(c) \leq l$ if $c$ is a linear combination of at most $l$ of the points $P e_{i}^{m}, 1 \leq i \leq m$. Let now $l$ be such that $r m / l \leq \delta / 2$. 
We see that every $x \in A_{E}^{\perp}(r)$ can be written as $x=b+c$ where $|b|_{2} \leq \delta / 2$ and $l(c) \leq l,|c|_{2} \leq 1$. Therefore we have

$$
N\left(A_{E}^{\perp}(r), \delta\right) \leq N\left(G_{l}, \delta / 2\right) \quad \text { where } \quad G_{l}=\left\{c \in \mathbb{R}^{m}: l(c) \leq l,|c|_{2} \leq 1\right\} .
$$

It is clear that $G_{l}$ can be covered by $\left(\begin{array}{c}m \\ l\end{array}\right)$ sets, each isometric to the $l$-dimensional euclidean unit ball. Therefore, by (4), we have

$$
N\left(G_{l}, \frac{\delta}{2}\right) \leq\left(\begin{array}{c}
m \\
l
\end{array}\right)\left(\frac{6}{\delta}\right)^{l} \leq \frac{m^{l}}{l !}\left(\frac{6}{\delta}\right)^{l} \leq\left(\frac{6 e m}{l \delta}\right)^{l},
$$

by Stirling's formula. Substituting $l=[2 \mathrm{rm} / \delta]+1$, we obtain (7), because $2 \mathrm{rm} / \delta \leq l \leq$ $3 \mathrm{rm} / \delta$.

The next theorem is a refinement of Kashin's splitting theorem (cf. [6, Corollary 6.4]). We think that it is of independent interest.

Theorem 3. Let $E \subset \mathbb{R}^{m}$ and $F \subset \mathbb{R}^{p}$ with $\operatorname{dim} E=\operatorname{dim} F=n$ and suppose $m \leq 3 n$ and $p \leq 3 n$. Then there is $Q \in O(F, E)$ such that

$$
\max \left\{|x|_{F}^{\perp},|Q x|_{E}^{\perp}\right\} \geq c|x|_{2} \quad \text { for all } x \in F .
$$

Moreover,

$$
v_{F, E}\left(\left\{Q: \max \left\{|x|_{F}^{\perp},|Q x|_{E}^{\perp}\right\} \geq c|x|_{2} \text { for all } x \in F\right\}\right) \geq 1-2^{-n} .
$$

Remark 1. Although conceptually close to Kashin's theorem, apparently this theorem neither implies nor is implied by it.

Remark 2. We have of course

$$
\max \left\{|x|_{F}^{\perp},|Q x|_{E}^{\perp}\right\} \geq(3 n)^{-1 / 2}|x|_{2} \quad \text { for all } x \in F ;
$$

the point is that the $c$ in Theorem 3 does not depend on $n$.

Proof of Theorem 3. Let $\delta, r$ be positive numbers to be determined later. Let

$$
B=\left\{T \in O(F, E):|T x|_{E}^{\perp} \geq \delta \text { for every } x \in A_{F}^{\perp}(r)\right\} .
$$

We see that if $T \in B$, then for every $x \in S_{F}$ we have

$$
\max \left\{|x|_{F}^{\perp},|T x|_{E}^{\perp}\right\} \geq \min (\delta, r) .
$$

Thus 9 holds with $c=\min (\delta, r)$ provided we can show that $v_{F, E}(B) \geq 1-2^{-n}$.

Let $N=N\left(A_{F}^{\perp}(r), \delta\right)$ and let $x_{1}, \ldots, x_{N} \in S_{F}$ be such that

$$
A_{F}^{\perp}(r) \subset \bigcup_{i=1}^{N} B\left(x_{i}, \delta\right) .
$$


Let us observe that $B \supset B^{\prime}$ where

$$
B^{\prime}=\left\{T \in O(F, E):\left|T x_{i}\right|_{E}^{\perp} \geq 2 \delta \text { for } i=1, \ldots, N\right\} .
$$

Indeed, suppose that $T \in B^{\prime}$. If $x \in B\left(x_{i}, \delta\right)$, then $T x \in B\left(T x_{i}, \delta\right)$, i.e. $T x=T x_{i}+y$ with $|y|_{2} \leq \delta$, hence

$$
|T x|_{E}^{\perp} \geq\left|T x_{i}\right|_{E}^{\perp}-|y|_{E}^{\perp} \geq 2 \delta-|y|_{2} \geq \delta .
$$

Hence, by [11], $|T x|_{E}^{\perp} \geq \delta$ for every $x \in A_{F}^{\perp}(r)$, thus $B \supset B^{\prime}$.

By (5) and (6), we have, for every $i$,

$$
v_{F, E}\left(\left\{T \in O(F, E): T x_{i} \in A_{E}^{\perp}(2 \delta)\right\}\right)=\sigma_{E}\left(A_{E}^{\perp}(2 \delta)\right) \leq(2 C \delta)^{n} .
$$

Therefore $v_{F, E}\left(B^{\prime}\right) \geq 1-N \cdot(2 C \delta)^{n}$, and thus, by (7),

$$
\nu_{F, E}\left(B^{\prime}\right) \geq 1-(3 e / r)^{3 r m / \delta} \cdot(2 C \delta)^{n} .
$$

Let now $r=\delta^{2}$ and let $\delta<1$ be such that

$$
2 C \delta \cdot\left(3 e / \delta^{2}\right)^{9 \delta} \leq 1 / 2 .
$$

Since $m \leq 3 n$, by (12), $v_{F, E}\left(B^{\prime}\right) \geq 1-2^{-n}$, thus, a fortiori, $v_{F, E}(B) \geq 1-2^{-n}$.

It will now be convenient to rephrase Theorem 3 in a setting of orthogonal transformations. Let us first observe that in the situation of Theorem 3, $Q$ determines $F$ and $E$. More precisely, $Q$ must be a partial isometry of rank $n$ from $\mathbb{R}^{m}$ to $\mathbb{R}^{p}$, i.e. a map of the form

$$
Q=\sum_{i=1}^{n} v_{i} \otimes u_{i},
$$

where $\left\{v_{i}\right\}_{i=1}^{n}$ is an orthonormal system in $\mathbb{R}^{m}$ and $\left\{u_{i}\right\}_{i=1}^{n}$ is an orthonormal system in $\mathbb{R}^{p}$. Then $F=Q^{*}\left(\mathbb{R}^{p}\right)$ and $E=Q\left(\mathbb{R}^{m}\right)$. We can therefore define

$$
\eta(Q)=\min _{x \in S_{F}} \max \left\{|x| \frac{\perp}{F},|Q x|_{E}^{\perp}\right\}
$$

with $F$ and $E$ as above.

Given an orthonormal system $v=\left\{v_{i}\right\}_{i=1}^{n}$ in $\mathbb{R}^{m}$, set $F=\operatorname{span}\left\{v_{1}, \ldots, v_{n}\right\}$ and define a map $Q(\cdot, v): O(p) \rightarrow O\left(F, \mathbb{R}^{p}\right)$ by

$$
Q(T, v)=\sum_{i=1}^{n} v_{i} \otimes T^{*} e_{i}^{n}
$$

Lemma 2. Let $m \leq 3 n$ and $p \leq 3 n$. Then for every orthonormal system $v=\left\{v_{i}\right\}_{i=1}^{n}$ in $\mathbb{R}^{m}$,

$$
v_{p}(\{T \in O(p): \eta(Q(T, v)) \geq c\}) \geq 1-2^{-n} .
$$


Proof. By the invariance properties of the measures $\nu_{F, E}$, for every measurable set $A \subset$ $O\left(F, \mathbb{R}^{p}\right)$ we have the following identities:

$$
\begin{gathered}
v_{p}(\{T \in O(p): Q(T, v) \in A\})=v_{F, \mathbb{R}^{p}}(A), \\
v_{F, \mathbb{R}^{p}}(A)=\int v_{F, E}(A \cap E) d E,
\end{gathered}
$$

where we integrate over the invariant probability measure on the Grassmannian of all $n$-dimensional subspaces of $\mathbb{R}^{p}$.

Let $A=\{T \in O(p): \eta(Q(T, v)) \geq c\}$. By $\left[9, v_{F, E}(A \cap E) \geq 1-2^{-n}\right.$ for every $E \subset \mathbb{R}^{m}$ with $\operatorname{dim} E=n$, and hence $(14)$ follows.

Given a sequence $\left\{T_{k}\right\}_{k=0}^{\infty}$ with $T_{k} \in O\left(3 \cdot 2^{k}\right)$ for $k=0,1, \ldots$, let us define, with the notation of (1):

$$
\begin{gathered}
E_{k}=\operatorname{span}\left\{u_{i}^{k}: i=1, \ldots, 2^{k}\right\}, \quad F_{k}=\operatorname{span}\left\{v_{i}^{k}: i=1, \ldots, 2^{k}\right\}, \\
Q_{k}=\sum_{i=1}^{2^{k}} v_{i}^{k} \otimes u_{i}^{k+1} .
\end{gathered}
$$

Lemma 3. Let $B_{n}=\left\{\left\{T_{k}\right\}: \eta\left(Q_{n}\right) \geq c\right\}$ for $n=1,2, \ldots$ Then

$$
v\left(B_{n}\right) \geq 1-2^{-2^{n}} .
$$

Proof. Given $\left(T_{1}, \ldots, T_{n-1}\right) \in \prod_{k=1}^{n-1} O\left(3 \cdot 2^{k}\right)$, let $v=\left(T_{n-1}^{*} e_{i}^{3 \cdot 2^{n-1}}\right)_{i=2^{n-1}+1}^{3 \cdot 2^{n-1}}$. Observe that $Q\left(T_{n}, v\right)=Q_{n}$, and therefore by (14),

$$
\nu_{3 \cdot 2^{n}}\left(\left\{T_{n} \in O\left(3 \cdot 2^{n}\right): \eta\left(Q_{n}\right) \geq c\right\}\right) \geq 1-2^{-2^{n}} .
$$

Integrating over all $\left(T_{1}, \ldots, T_{n-1}\right) \in \prod_{k=1}^{n-1} O\left(3 \cdot 2^{k}\right)$ gives 15, , since $B_{n}$ does not depend on $T_{n+1}, T_{n+2}, \ldots$

Let now $A_{n}=\left\{\left\{T_{k}\right\}: \eta\left(Q_{k}\right) \geq c\right.$ for $\left.k=n, n+1, \ldots\right\}=\bigcap_{k \geq n} B_{k}$. By [15,

$$
v\left(A_{n}\right) \geq 1-2 \cdot 2^{-2^{n}}
$$

Lemma 4. For every $\left\{T_{k}\right\} \in A_{n}, Y_{q}\left(\left\{T_{k}\right\}\right)$ is isomorphic to $\ell_{q}$ for all $1<q \leq 2$.

This lemma clearly implies Theorem 2.

Proof. By 10, , for every $\left\{T_{k}\right\} \in A_{n}$,

$$
\eta\left(Q_{k}\right) \geq c^{\prime}=\min \left\{c,\left(3 \cdot 2^{n}\right)^{-1 / 2}\right\} \quad \text { for every } k=1,2, \ldots
$$

This means that for every $v \in F_{k}$, and all $k=1,2, \ldots$,

$$
\max \left\{|v|_{F_{k}^{\perp}},\left|Q_{k} v\right|_{E_{k+1}}^{\perp}\right\} \geq c^{\prime}|v|_{2} .
$$


Let

$$
\begin{aligned}
& E=\left\{\left(0, a_{1}, a_{2}, \ldots\right): a_{k} \in E_{k}, k=1,2, \ldots\right\} \subset X, \\
& F=\left\{\left(b_{0}, b_{1}, b_{2}, \ldots\right): b_{k} \in F_{k}, k=0,1,2, \ldots\right\} \subset X,
\end{aligned}
$$

both equipped with the norm $\|\cdot\|_{2, q}$. It is clear that $Q$ defined by

$$
Q\left(b_{0}, b_{1}, b_{2}, \ldots\right)=\left(0, Q_{0} b_{0}, Q_{1} b_{1}, \ldots\right)
$$

is an isometry of $F$ onto $E$ and that their $\|\cdot\|_{2, q}$-completions are isometric to the $\ell_{q}$-sum of $\ell_{2}^{2^{k}}, k=0,1,2, \ldots$, hence isomorphic to $\ell_{q}$. We just need to exhibit an isomorphism $S$ from $Y_{q}$ onto the $\|\cdot\|_{2, q}$-completion of $F$.

Every $x \in Y$ has a unique representation

$$
x=\sum_{k=0}^{\infty}\left(v^{k}+u^{k+1}\right)
$$

where $v^{k} \in F_{k}, u^{k+1} \in E_{k+1}$ and evidently $u^{k+1}=Q_{k} v^{k}$. Let

$$
v=\sum v^{k}, \quad u=\sum u^{k+1} .
$$

Define $T x \in F$ by $T x=\left(v^{0}, v^{1}, v^{2}, \ldots\right)$. It is clear that $T$ is a surjective map from $Y$ onto $F$. We will show that

(*) $2\|T x\|_{2, q} \geq\|x\|_{q, q}$,

(**) $\left(c^{\prime} / 4\right)\|T x\|_{2, q} \leq\|x\|_{q, q}$,

thus $T$ can be extended to an isomorphism $S$ of $Y_{q}$ onto the $\|\cdot\|_{2, q}$-completion of $F$.

For $(*)$ we have $x=u+v$, thus

$$
\|x\|_{q, q} \leq\|u\|_{q, q}+\|v\|_{q, q} \leq\|u\|_{2, q}+\|v\|_{2, q}=2\|v\|_{2, q}=2\|T x\|_{2, q} .
$$

For $(* *), x$ has a unique representation $x=\sum x^{k}$ with $x^{k} \in \mathbb{R}^{3 \cdot 2^{k}}$ (to wit $x^{k}=$ $v^{k}+u^{k}$ for $\left.k \geq 1, x^{0}=v^{0}\right)$. We have, for $k \geq 1$,

$$
\begin{aligned}
\left|x^{k}\right|_{q}^{q}+\left|x^{k+1}\right|_{q}^{q} & =\left|v^{k}+u^{k}\right|_{q}^{q}+\left|u^{k+1}+v^{k+1}\right|_{q}^{q} \\
& \geq \frac{1}{2}\left(\left|v^{k}+u^{k}\right|_{q}+\left|u^{k+1}+v^{k+1}\right|_{q}\right)^{q} \\
& \geq \frac{1}{2}\left(\left|v^{k}+u^{k}\right|_{1}+\left|u^{k+1}+v^{k+1}\right|_{1}\right)^{q} \geq \frac{1}{2}\left(\left|v^{k}\right|_{F_{k}}^{\perp}+\left|u^{k+1}\right|_{E_{k+1}}^{\perp}\right)^{q} \\
& =\frac{1}{2}\left(\left|v^{k}\right|_{F_{k}}^{\perp}+\left|Q_{k} v^{k}\right|_{E_{k+1}}^{\perp}\right)^{q} \geq \frac{\left(c^{\prime}\right)^{q}}{2}\left|v^{k}\right|_{2}^{q},
\end{aligned}
$$


by 16. Summing over $k$ we obtain

$$
\begin{aligned}
\|x\|_{q, q}^{q} & =\sum_{k=0}^{\infty}\left|x^{k}\right|_{q}^{q}=\left|v^{0}\right|_{q}^{q}+\frac{1}{2}\left(\sum_{k=1}^{\infty}\left|x^{k}\right|_{q}^{q}+\sum_{k=1}^{\infty}\left|x^{k}\right|_{q}^{q}\right) \\
& \geq \frac{1}{3}\left|v^{0}\right|_{2}^{q}+\frac{1}{2} \sum_{k=1}^{\infty}\left(\left|x^{k}\right|_{q}^{q}+\left|x^{k+1}\right|_{q}^{q}\right) \geq \frac{1}{3}\left|v^{0}\right|_{2}^{q}+\frac{\left(c^{\prime}\right)^{q}}{4} \sum_{k=1}^{\infty}\left|v^{k}\right|_{2}^{q} \\
& \geq \frac{\left(c^{\prime}\right)^{q}}{4} \sum_{k=0}^{\infty}\left|v^{k}\right|_{2}^{q}=\frac{\left(c^{\prime}\right)^{q}}{4}\|T x\|_{2, q}^{q} .
\end{aligned}
$$

This proves Lemma 4 and Theorem 2.

A historical remark. The original argument that the "Enflo-Davie spaces" $Y_{p}$ fail to have AP for $2<p<\infty$ evidently breaks down for $1 \leq p<2$ (otherwise it would imply that $Y_{2}$, which is a Hilbert space, does not have AP). However, it has not been clear whether another approach would imply that $Y_{p}$ fails to have AP for $1 \leq p<2$. Here we have shown that this is not the case: at least "generically", $Y_{p}$ does have AP for $1 \leq p<2$.

Remark. We do not know whether the roles of $p$ and $q$ in the abstract of this paper can be reversed. However, for $X=\ell_{\infty}$ an answer is known by the results of [1]: if $X$ is an $\mathcal{L}_{\infty}$ space and if $Y \subset X$ has BAP, then $X / Y$ has BAP as well (cf. also [5]). "Interpolating" between $\ell_{\infty}$ and $\ell_{2}$, this suggests the following question:

Question. Let $2<p<\infty$, and let $Y$ be a subspace of $\ell_{p}$ which has BAP. Does $\ell_{p} / Y$ necessarily have BAP?

Acknowledgments. The author wants to express his thanks to Bill Johnson for turning his attention to the problem and for numerous helpful discussions.

The author was supported in part by EU/FP6 Marie Curie ToK SPADE2.

\section{References}

[1] Figiel, T., Johnson, W. B., Pełczyński, A.: Some approximation properties of Banach spaces and Banach lattices. To appear

[2] Johnson, W. B., Rosenthal, H. P.: On $w^{*}$-basic sequences and their applications to the study of Banach spaces. Studia Math. 43, 77-92 (1972) Zbl 0213.39301 MR 0310598

[3] Lindenstrauss, J.: On James's paper "Separable conjugate spaces". Israel J. Math. 9, 279-284 (1971) Zbl 0216.40802 MR 0279567

[4] Lindenstrauss, J., Tzafriri, L.: Classical Banach Spaces I, Sequence Spaces. Springer, Berlin (1977) Zbl 0362.46013 MR 0500056

[5] Lusky, W.: A note on Banach spaces containing $c_{0}$ or $C_{\infty}$. J. Funct. Anal. 62, 1-7 (1985) Zbl 0581.46016 MR 0790767

[6] Pisier, G.: The Volume of Convex Bodies and Banach Space Geometry. Cambridge Tracts in Math. 94, Cambridge Univ. Pres (1989) Zbl 0933.46013 MR 1036275

[7] Schechtman, G.: Special orthogonal splittings of $L_{1}^{2 k}$. Israel J. Math. 139, 337-347 (2004) Zbl 1066.46010 MR 2041797 